\title{
Globalización y medios de comunicación: carencias sociales de los efectos económicos
}

\author{
Sara RoBlas MARTíN*
}

Propuesto: 7 de marzo de 2012

Evaluado: 9 de abril de 2012

Aceptado: 6 de mayo de 2012

(Abstracts y palabras clave al final del texto)

La vida internacional, cada día más intensa, no suprime la vida de las naciones, la vida de las ciudades, la vida de las aldeas: se superpone a ellas, y todos nosotros experimentamos, en los rincones más recónditos de nuestros respectivos territorios, la repercusión de los acontecimientos que ocurren fuera de sus fronteras.

(Mattelart. 2006:26)

\section{PERSPECTIVAS SOBRE GLOBALIZACIÓN Y MUNDIALIZACIÓN}

A la hora de definir un marco que establezca las perspectivas desde las que pretendo acercarme al fenómeno de la globalización y sus efectos tanto en el aspecto mediático como en el social, el carácter transversal y la complejidad del término me anima a delimitar el espectro desde el que voy a abordarlo, teniendo en cuenta distintos ámbitos de actuación. Por ello, a lo largo del artículo centro mi atención principalmente en cuatro autores que exponen su visión de este fenómeno desde distintas líneas de actuación. En primer lugar, Amartya Sen ${ }^{1}$, teniendo en cuenta sus planteamientos económicos y su especialidad en el ámbito del desarrollo. Por otro lado David Held de acuerdo a las líneas que dibuja en lo que respecta a sus conocimientos en el ámbito de la sociología y en las relaciones internacionales ${ }^{2}$. Para centrarme en la perspectiva política y destacar factores relacionados con instituciones supranacionales, me baso en las aportaciones del Secretario General de las Naciones Unidas

* Investigadora y doctoranda Universidad Complutense de Madrid

1 Premio Nobel de Economía en 1998, ha desarrollado numerosas investigaciones, estudios y análisis sobre la globalización y sus efectos en las sociedades, especialista en el ámbito del desarrollo y la pobreza a nivel mundial.

2 Principalmente a través de su obra Un pacto global (2005). 
de 1997 a 2006, Kofi Annan, especialmente en lo que se refiere a la Declaración del Milenio. Por su parte, los análisis y estudios realizados por Armand Mattelart permiten aproximarse a conceptos como mundialización, sociedad de la información y nuevo orden mundial de la comunicación. A pesar de que cada autor se aproxima al fenómeno de la globalización desde una perspectiva distinta, todos coinciden en mantener al ser humano como principio, centro y fin de cualquier proceso que se realice a nivel mundial.

La globalización es un concepto que se ha empleado en la mayoría de las ocasiones como sinónimo de mercado internacional de acuerdo a las líneas trazadas desde el $\mathrm{FMI}^{3}$, la Comisión Europea ${ }^{4}$ y el Banco Mundial ${ }^{5}$. Por ello, cuando se emplea el término globalización, el contenido intrínseco de dicho concepto está fuertemente ligado a factores económicos. De esta forma, la prioridad economicista eclipsa cualquier otra variable que, de forma lógica y consecuente, esté vinculada al propio sentido de la globalización como son los aspectos sociales que se ven afectados directamente por los efectos económicos, positiva o negativamente. Es lo que Mattelart denomina capitalismo integrado ya que se trata de lograr un aumento de la competitividad a escala mundial. Según Mattelart la globalización "trata de abarcar el proceso de unificación del campo económico, y, por extrapolación, de diagnosticar la situación general del mundo." (2007:85). Nos situamos pues en un espacio de intercambio basado en el mercado, donde las relaciones, vinculaciones y acciones que se realicen parten de la obtención de un beneficio. Sin embargo, este

3 Definición dada por el Fondo Monetario Internacional: "La globalización se refiere a la creciente dependencia económica mutua entre los países del mundo ocasionada por el creciente volumen y variedad de transacciones transfronterizas de bienes y servicios, así como por la de flujos internacionales de capitales, y por la aceleración de la difusión de la tecnología en más lugares del mundo. Trae consigo nuevas oportunidades y nuevos desafíos para las economías y los rectores de la política económica. En general, las ventajas que ofrece la globalización son en esencia semejantes a las que atribuían los economistas clásicos a la especialización y a la ampliación de los mercados a través del comercio. La globalización eleva la productividad y el nivel de vida promedio ya que incrementa la división internacional del trabajo y la eficiencia en la asignación del ahorro y, al tener los consumidores mayor acceso a los productos extranjeros, pueden disfrutar de una mayor oferta de bienes y servicios a precios inferiores. La globalización también ofrece otros beneficios: por ejemplo, puede permitir que un país movilice un mayor volumen de ahorro financiero (ya que los inversionistas tienen acceso a una mayor variedad de instrumentos financieros en diferentes mercados) y aumentar la competencia entre las empresas." Definición recogida en "Perspectivas de la economía mundial", Estudios Económicos y Financieros, Fondo Monetario Internacional, Washington, marzo 1997, p.50.

4 La Comisión Europea se refiere a la globalización como "el proceso mediante el cual los mercados y la producción de diferentes países están volviéndose cada vez más interdependientes debido a la dinámica del intercambio de bienes y servicios y a los flujos de capital y tecnología. No se trata de un fenómeno nuevo, sino de la continuación de desarrollos que habían estado funcionando durante un tiempo considerable". Citado por Grahame Thompson en "Introducción: para situar la globalización", Revista Internacional de Ciencias Sociales, 160, junio 1999, p. 2.

5 El Banco Mundial habla de la globalización como "un cambio general que está transformando a la economía mundial, un cambio que se refleja en vinculaciones internacionales cada vez más amplias e intensas del comercio y las finanzas y el impulso universal hacia la liberación del comercio y los mercados de capital por la creciente internacionalización, y por un cambio tecnológico que está erosionando con rapidez las barreras que obstaculizan el comercio internacional de bienes y servicios y la movilidad del capital". Citado por Klauss Schaeffler en "Las empresas multinacionales en América Latina ¿Promotoras del desarrollo o villanos de la globalización?", en Revista Contribuciones, 3, Konrad Adenauer Stiftung - CIEDLA, Buenos Aires, julio-septiembre 1998, p. 107. 
intercambio no conlleva que dicho beneficio sea ni común ni equitativo. El principio de los mercados es la búsqueda del máximo beneficio; una estructura social que basa su existencia en la mera participación en los mercados produce efectos devastadores ${ }^{6}$. Si el único foro de intercambio en el que es posible participar es el del mercado, las sociedades se transforman en agentes económicos que ofrecen o adquieren lo que consideren oportuno con el objetivo de conseguir el máximo beneficio, no de solventar los problemas de los miembros de su propia sociedad ni de conseguir el bien común. De este modo, las sociedades comienzan a funcionar como si de empresas se tratara. Si éstas no resultan rentables o no son competitivas, desaparecen del panorama internacional. Sin embargo, hay una enorme diferencia entre la idea de desaparición de una empresa y la de una sociedad en el ámbito global: la primera puede reagruparse o simplemente desaparecer sin mayores consecuencias más que pérdidas económicas; por su parte, las sociedades aunque desaparezcan del panorama internacional, no dejan de existir sino que se ven sometidas al aislamiento, la absorción o la supeditación de otras sociedades con mayor poder económico que finalmente serían las que tomaran las decisiones por ellas.

La pretensión de que la única forma de actuar globalmente es a través del comercio y la búsqueda del beneficio económico, obvia factores como la sanidad, la educación, los derechos civiles, la participación social, la comunicación o la información, empobreciendo a las sociedades. En este sentido solo las zonas o los actores internacionales con mayor poder comercial podrían operar libremente, estableciendo normas, y ejerciendo el control sobre otras zonas con menos capacidades que quedarían excluidas o sometidas. Este tipo de tratamiento ha puesto en entredicho actuaciones que parten del concepto de globalización en su carácter meramente económico. La idea de expansión de influencias y mercados es una mala interpretación de los fenómenos que derivan de procesos mundiales de intercambio.

Si partimos de la idea de que toda sociedad tiene una estructura y una complejidad propias, es necesario admitir que, al mismo tiempo, es también incompleta y que carece de aspectos que otras sociedades, con otras estructuras y complejidades, sí que poseen. A partir de esta premisa cualquier intercambio tendría que ser llevado a cabo en condiciones de igualdad y respeto, y el resultado sería el enriquecimiento social de ambos conjuntos sociales. La imbricación de determinados aspectos no significa la sumisión ni el sometimiento de una sociedad a otra ni tampoco la renuncia de características propias. El valor y características que cada sociedad tiene y que le son inherentes, le confiere y otorga un reconocimiento a nivel mundial.

Las perspectivas pesimistas respecto a los procesos de globalización se fundamentan precisamente en su carácter exclusivamente economicista. Paul Collier se

6 En su artículo "Globalización y pobreza", Vandana Shiva expone casos concretos en los que los mercados internacionales han abatido a pequeñas sociedades de la zona del Punjab. Shiva afirma que: "son experiencias como estas las que me han enseñado que estamos muy equivocados con respecto a la economía global y que es preciso detenernos a pensar acerca del impacto de la globalización sobre la vida de la gente común. Esto es vital para alcanzar la sustentabilidad”. Dicho artículo fue publicado en la Revista Web Mensual de Economía, Sociedad y Cultura, en mayo de 2000. Disponible en el siguiente enlace: http://rcci.net/globalizacion/2000/fg126.htm. 
posiciona en la convicción de que la globalización arrastrará negativamente a países con pocos recursos que se verán condenados a la dependencia:

¿Será capaz la globalización de mejorar el panorama? Ya hemos analizado cómo afectará ésta a los países del furgón de cola. Es muy posible que el comercio los hunda en la dependencia de recursos naturales y muy poco probable les abra nuevas oportunidades, como también es más probable que la movilidad internacional de la mano de obra cualificada, en lugar de servirles de motor de crecimiento, les arranque el poco capital y talento que tienen (2009:285).

Por su parte Amartya Sen ${ }^{7}$ dibuja dos perspectivas frente a los procesos de globalización. Sen afirma que las posturas contrarias se basan en que la globalización provoca que los picos de pobreza y riqueza sean cada vez más extremos. Por otro lado, los defensores de la globalización alegan que los países que son capaces de entrar en el intercambio global, ven reducida su pobreza. El problema estriba en que la mayor parte de los países empobrecidos no tienen la capacidad, ni por tanto la oportunidad, de acceder al mercado global, por lo que si solo se atiende a los que pueden participar en él, se está excluyendo a millones de personas. De esta forma se estaría cayendo en una exclusión desigual de acuerdo a la capacidad de cada zona o lo que Klisksberg y Sen denominan la "exclusión de la inclusión equitativa" (2007:29).

La forma de evitar este hecho sería a través de la puesta en práctica de cambios radicales en las políticas internas (en las que se destinaran más recursos para la educación básica, la salud y los microcréditos) y de las políticas internacionales, en especial de los países ricos (a través de la reducción de aranceles a los productos agrícolas, textiles e industriales de los países pobres, condonando la deuda externa y promoviendo programas de desarrollo). Sin embargo, es necesario tener en cuenta que aunque los países con menos recursos pudieran participar en la economía global e intercambiar sus productos, esto no aseguraría que recibiesen una parte justa de los beneficios. Por tanto, lo importante es plantearse alternativas globales que beneficiasen más a todos a través de nuevos acuerdos nacionales e internacionales.

Los procesos globales solo pueden ser entendidos en un sistema equilibrado en el que los países que aporten al conjunto se vean beneficiados por dicha aportación; consistiría pues en un intercambio equitativo en el que la mejora obtenida fuera global y en la que no se perdiera la perspectiva de ser humano frente a la de sujeto de consumo. Es así como surge el concepto de mundialización, que profundiza más en factores sociales prestando atención a los cambios humanos. La mundialización sería definida como un nuevo contexto y cauce en el que se atiende especialmente a la consecución de objetivos sociales, laborales, de medio ambiente, relativos a los derechos humanos y a la reducción de la pobreza. Este tipo de actitudes son las que llevan a la integración, a la igualdad y a la priorización del individuo, de las sociedades y de sus necesidades frente a la búsqueda del beneficio económico y empresarial que puedan perseguir las grandes multinacionales.

\footnotetext{
7 En su artículo "Pobreza global, justicia global", Sen plantea los diferentes argumentos que son defendidos por los opositores y defensores de la globalización.
} 
[...] la palabra "mundialización" [...] se trata de un término que se refiere explícitamente a la filosofía holística, esto es, a la idea de una unidad totalizadora o unidad sistémica. Una empresa global es una estructura orgánica en la que cada parte debe servir a la totalidad. Cualquier fallo en la "interoperabilidad" entre las partes, cualquier obstáculo al libre intercambio de los flujos, trae el riesgo de colapsar el sistema. La comunicación ha de mantenerse omnipresente (Mattelart, 2007:87).

Por otro lado, hay un aspecto importante a la hora de hablar de globalización, y es la influencia que unas zonas ejercen sobre otras. La consideración de que los planteamientos occidentales han sido los adalides de los procesos de globalización actual, ha creado recelos, incertidumbres y en ocasiones temores ante la pérdida de la identidad.

A la globalización se la considera con frecuencia como igual a occidentalización global. Tanto quienes la favorecen como quienes son contrarios suelen compartir este criterio. Quienes tienen una óptica muy positiva de la globalización la consideran como una maravillosa contribución de la civilización occidental al mundo. [...] Desde la perspectiva opuesta, el dominio de occidente -considerado algunas veces como una continuación del imperialismo occidental- es el malo de la película. Según esta óptica, el capitalismo contemporáneo, impulsado y conducido por países codiciosos y voraces de Europa y Norteamérica, ha establecido reglas comerciales y relaciones de negocios que no son favorables para los intereses de los pueblos más pobres del mundo." (Kliksberg y Sen, 2007:13).

No obstante, $\operatorname{Sen}^{8}$ defiende la idea de que la globalización no es sinónimo de occidentalización. Los que ante esta afirmación se muestran optimistas consideran que Occidente ha aportado mucho al desarrollo social, económico y cultural, por lo que cualquier aspecto que globalice será bueno para la humanidad. Los que opinan lo contrario consideran que Occidente sólo ha ejercido dominación a través del imperialismo occidental. De esta forma el capitalismo ha impuesto reglas comerciales que privan o excluyen a los países pobres.

Por su parte, la argumentación que aporta Held (2005) es la de que globalización no es lo mismo que americanización y no tiene porqué ser occidental. Afirma que no va ligado al fin de Estado-nación y no constituye una amenaza para las culturas nacionales. Held destaca principalmente la posibilidad que ofrece respecto a la apertura mundial, especialmente en el ámbito de la comunicación. Un mundo interconectado parte de la consideración de que existen asuntos que son abordados desde esferas políticas delimitadas (vivienda, educación, orden), pero otros factores como la salud mundial, la economía global o el medio ambiente, precisan la creación de instituciones nuevas y más extensas.

El establecer un sistema político socialdemócrata global, debe estar fundamentado en estados y organismos comprometidos con los principios democráticos y los derechos humanos. Este tipo de sistema político precisaría establecer un marco tota-

8 En su artículo "Juicios sobre la globalización", analiza las distintas concepciones que existen sobre la globalización. Para ello recoge opiniones dispares, centrándose en la idea de la supuesta pertenencia de dicho proceso por parte de comunidades occidentales. 
lizador de foros públicos democráticos que abarcase ciudades, estados-nación, regiones y, por último, el conjunto internacional. En el ámbito local se podría establecer un proceso participativo y directo, mientras que en el global se necesitarían procesos representativos. La importancia de destacar factores como Estado de derecho, igualdad política, sistema democrático, justicia, solidaridad social y eficiencia económica, es imprescindible para establecer un sistema de interconexión global, lo que Held denomina "socialdemocracia global" (2005:40). Si se parte de estas ideas es posible construir un espacio internacional en el que la transparencia, la rendición de cuentas o el compromiso con la justicia social sean las pautas que lo determinen.

Sin embargo, las dificultades reales a las que se enfrentan la globalización y la mundialización retrasa o impide el desarrollo de todas las expectativas que este tipo de procesos pueden ofrecer. Por ello, el esfuerzo debe ser aún mayor desde estructuras y sistemas supranacionales. Es así cómo Kofi Annan, en la Asamblea General de Naciones Unidas de 2000, afirmaba que

no hay vocación más noble ni responsabilidad mayor que la de facilitar los medios a hombres, mujeres y niños, en las ciudades y pueblos de todo el mundo, para que puedan vivir mejor. Sólo cuando esto ocurra sabremos que la mundialización está empezando de verdad a incluir a todos para que todos compartan las oportunidades que ofrece" $"$.

En este contexto es en el que las Naciones Unidas establecen su labor, su implicación y su compromiso con las acciones globales en los procesos de mundialización, tratando de conseguir tres objetivos: un mundo sin miseria, un mundo sin temor y un futuro sostenible.

\section{LOS MEDIOS COMO ACTORES Y CONSTRUCTORES INTRÍNSECOS DE LAS SOCIEDADES}

La idea de aldea global desarrollada por MacLuhan ${ }^{10}$ en la década de los 60 , sitúa a los sistemas de información y comunicación en factores clave en las relaciones internacionales y mundiales. Los medios de comunicación adquieren un papel social primordial, ya que actúan como informadores de los ciudadanos ante cualquier intercambio o acción que realicen tanto los gobiernos, como organismos internacionales, supranacionales o las distintas sociedades a nivel mundial. Son los que facilitan la adquisición de conocimiento global a las zonas locales. Son portadores y responsables de muchos de los intercambios a nivel global, y a través de ellos es posible construir y establecer sistemas de comunicación e información accesibles a todos. Uno de los factores que ha favorecido en los últimos tiempos la aceleración

\footnotetext{
9 Informe del Secretario de Naciones Unidas en la Asamblea General dedicada al Milenio: "Nosotros, los pueblos: la función de las Naciones Unidas en el siglo XXI". 27 de marzo de 2000. Disponible en el siguiente enlace: http:/unpan1.un.org/intradoc/groups/public/documents/un/unpan004567.pdf

10 El concepto "aldea global" aparece en sus obras Gutenberg Galaxy: The Making of Typographic Man (1962), Understanding Media (1964) y Guerra y paz en la Aldea Global (1968).
} 
de los procesos de globalización, ha sido principalmente la creación de diversos sistemas de comunicación que han permitido interconectar poblaciones de forma instantánea manteniendo cada vez más la fiabilidad de las informaciones. Este gran avance es lo que ha llevado al extremo el proceso de globalización que comenzó cuando se produjeron los primeros intercambios a gran escala entre los pueblos.

La comunicación siempre debe ser entendida como el medio supremo de interacción del ser humano; no puede convertirse en un problema de base, sino ser la que ofrezca posibles soluciones a los problemas básicos. Por ello, su espacio en las relaciones internacionales es fundamental. Es importante tener presente que la comunicación en origen parte del individuo para ir evolucionando a los ámbitos comunitario, internacional y finalmente mundial. No tendría sentido partir de una comunicación general y planetaria sino se considerara al ser humano como ciudadano, como parte activa y constituyente de la propia realidad en la que está inmerso, en continua interrelación con el mundo que le rodea. Por su parte el individuo ha de ser consciente de su propia realidad y de la necesidad que tiene de interactuar en ella para lograr su propio beneficio y el del conjunto.

En este proceso la comunicación que se realice es fundamental, ya que cualquier acto de comunicación implica una receptividad y una respuesta. En el ámbito de la cooperación los procesos de comunicación son complejos y se mueven en múltiples líneas todas ellas esenciales para la buena consecución de resultados. Para ello, es necesario que la información parta del país o la zona en la que se va a trabajar, de forma que se puedan conocer todos los aspectos relacionados con la sociedad que la ocupa y se pueda colaborar de forma coherente, intensa, estable y duradera. A pesar de que las nuevas tecnologías han abierto múltiples vías de intercambio y apertura, cuando el acceso a los medios se convierte en un obstáculo para zonas empobrecidas, la cooperación para el desarrollo cobra aún más relevancia. Surgen así nuevas perspectivas y nuevos modelos de acuerdo a las necesidades que exigen una renovación de estructuras que atiendan de forma más directa y fiel a los problemas que afectan a las sociedades, impulsando su participación.

Los llamativos debates sobre las ayudas urgentes han ocultado, sin embargo, otras rupturas más discretas que se produjeron en las relaciones entre sociedades civiles. Aparecerán nuevas formas de "cooperación descentralizada" y de redes de intercambios recíprocos de conocimientos, que se verán impulsadas por una filosofía del desarrollo que pone en tela de juicio los términos del intercambio. El modelo difusionista de comunicación persuasiva es sustituido por una reflexión sobre la "democracia participativa" y sobre el papel que desempeñan los diversos medios de comunicación. (Mattelart, 2007:82)

Si partimos de la importancia que adquieren en nuestros días los procesos de globalización y mundialización como multiplicadores de acciones económicas, comunicativas y sociales, los medios de comunicación desarrollan una función primordial. Son el mejor ejemplo de actuaciones globales, ya que constituyen el resultado de largos procesos de intercambio entre diversas sociedades. La evolución que han experimentado los medios, se mueve en consonancia con las necesidades de interconexión que van surgiendo a nivel mundial en la búsqueda de soluciones a pro- 
blemas globales. Para que realmente cualquier intercambio de este tipo produzca un resultado positivo y beneficioso para todas las partes intervinientes, es necesario que se parta de la idea de equilibrio e igualdad. Ambos conceptos descartan la adaptación o supeditación que en ocasiones suele suceder cuando zonas con diversas capacidades comparten un proyecto común.

Por ello, los sistemas de información de los distintos países necesitan adoptar una cierta unificación de los lenguajes para poder llevar a cabo acciones efectivas y eficaces. Cuando se comparten las mismas herramientas y vías de difusión se simplifican procesos que de otra forma se volverían complejos ralentizando y obstaculizando el objetivo final. Si se busca una difusión rápida, estable y al alcance de todos es necesario compatibilizar y agrupar patrones. Sin embargo, la facilidad y rapidez con que se difunda la información no es el único factor que afecta a los procesos de globalización en los medios. La forma de difundir el mensaje, las informaciones que se transmitan y prioricen son las claves donde surgen los conflictos y problemas de comunicación global. Y es ahí donde la unificación que puede producirse por medio de las agencias y empresas informativas se vuelve un error si se concibe como la existencia de una única vía y forma de comprensión de la información recibida por parte de los pueblos. El factor mundial es complejo y no solo le afectan aspectos unilaterales ni unidireccionales; la información parte de múltiples puntos que pueden incluso interconectarse unos con otros, y a medida que ampliamos las fronteras comunicativas aumentamos el abanico de posibilidades, las formas de comprensión y las concepciones del mundo. Es por ello esencial partir del conocimiento real, multidisciplinar y complejo de las distintas realidades, tratando de plasmar diferentes puntos de vista, ya que patrones simplistas llevan a concepciones simplistas y por tanto, a errores de comprensión y de comunicación. Ejemplo de ello, es el programa llevado a cabo por la asociación india de medios comunitarios "Namma Dhwani" ("Nuestras Voces" en lengua kannada), formada por miembros de la comunidad de Budikote, las ONG Voices y Myrada y el apoyo de la UNESCO. Tras analizar la situación mediática en la que se encontraba la zona en 1999 quedó de patente la falta de un sistema mediático que informara a sus habitantes de los cultivos, los precios del mercado o aspectos relacionados con la salud. Debido a los altos niveles de pobreza y analfabetismo de los habitantes de la zona, los medios impresos quedaban fuera de su alcance, y por ello sus habitantes buscaban la información en medios radiofónicos que difundieran en su lengua. Gracias a la creación de medios comunitarios por parte de esta asociación, el vacío informativo existente fue cubierto, favoreciendo al desarrollo pleno de esta zona, ya que el medio se ajustaba a las necesidades del lugar ${ }^{11}$.

Cuando se actúa de esta forma y se potencia la diversidad se está apostando por la fidelidad a la realidad y el entendimiento de que tras las informaciones están las sociedades que las construyen y a las que les afecta. Considerar que informaciones pertenecientes a pequeños grupos pueden carecer de interés para el conjunto, obliga a redundar en informaciones globales y a reducir las ideas a concepciones genera-

11 Puede consultarse el proyecto en el siguiente enlace del Programa Internacional para el Desarrollo de la Comunicación (PIDC): http://www.unesco-ci.org/ipdcprojects/content/namma-dhwani-community-radio 
les, superficiales que pueden llevar al error. Bien es cierto que al difundir información, ésta se organiza de acuerdo a una priorización (agenda setting) en consonancia con el sistema de valoración y ponderación que tenga cada agencia o empresa de información. Sin embargo, cuando estas valoraciones se elevan al ámbito global, las consideraciones que hay que tener presentes deben permitir un conocimiento más profundo y fiel de las distintas realidades. De esta forma la necesidad imperiosa de priorizar contenidos, no perjudicaría a informaciones que de otro modo quedarían ocultas. La presencia de informaciones de países con un bajo índice de Desarrollo Humano (IDH) ${ }^{12}$ suele ser menor en proporción que la de zonas con un alto IDH. Anwarul Chowdhury, miembro del alto comisionado de Naciones Unidas, afirmaba en la presentación del Pacto Mundial de los medios de comunicación que:

es lamentable que la voz de los países menos desarrollados continúe marginada. Existe una necesidad de aumentar la atención en los retos que afrontan día tras día millones de personas pobres en estas naciones. Sus necesidades deben ser prioritarias en la agenda de desarrollo mundial ${ }^{13}$.

Esta desproporción en cuanto a cantidad de información distribuida suele corresponderse también con el contenido de dicha información, por lo que encontramos que en muchas ocasiones zonas empobrecidas quedan excluidas y supeditadas a lo que desde zonas con mayor nivel económico se determine. Para que estos desequilibrios no se produzcan es preciso buscar en todo momento un intercambio enriquecedor entre factores distintos y dispares sin que se pierda en ningún momento la identidad por alguna de las partes. La importancia de preservar la identidad deriva de la pertenencia de cada individuo con la sociedad en la que se desarrolla, lo que no resta que mantenga relaciones de intercambio a nivel internacional. Amartya Sen (2007:65) recogía lo que ya señaló Michael Sendel sobre la importancia de la comunidad para el individuo y la relación intrínseca que existe entre ambos:

La comunidad describe no solo lo que tienen sus miembros como conciudadanos, sino también lo que son, no una relación que escogen (como en una asociación voluntaria), sino un apego que descubren, no simplemente un atributo sino algo que constituye su identidad 14 .

\footnotetext{
12 Son medidores estadísticos estandarizados basados en múltiples variables a través de los que se pretende establecer un baremo para poder comparar el nivel de desarrollo de los diversos países. Mediante tres parámetros (vida larga y saludable, educación y nivel de vida digno), el IDH trata de aproximarse a la realidad de las distintas sociedades teniendo en cuenta aspectos no cuantificables. Para calcular el IDH se tiene en cuenta el INB per cápita (Ingreso Nacional Bruto) en vez del PIB per cápita (Producto Interior Bruto). El Ingreso Nacional Bruto (INB) es el valor de todos los bienes producidos por los miembros residentes de un país durante un año dividido entre el número de habitantes totales de ese país. Para ello emplea el Método Atlas y los valores de Paridad de Poder Adquisitivo (PPA) per cápita a través del cual se puede comparar de una forma más realista y ajustada el nivel de vida que tiene cada país.

13 Presentación del "Pacto Mundial de los medios de comunicación", propuesta realizada por Naciones Unidas para promover la presencia de contenidos relacionados con el desarrollo en las empresas informativas locales e internacionales, presentada en octubre de 2006, en Nueva York.

14 Véase: Sendel, M. (1998): Liberalism and the limits of justice. Reino Unido: Cambridge Univesity Press, pp.150-151.
} 
En este mismo escenario, hay que tener en cuenta que el desarrollo de las nuevas tecnologías de la información y la comunicación a pesar de que por un lado reducen distancias, por otro aumentan las diferencias. Es lo que se llamó la brecha digital ${ }^{15}$. En este sentido surge el peligro de que zonas con más capacidad adquisitiva acaparen el dominio y monopolio de las fuentes de información, estableciendo sistemas mundiales controlados desde su perspectiva. Por ello, cualquier postura, acción o sistema que permita o busque la reducción de posibilidades de zonas con menos recursos o su esclavización a sistemas con mayores capacidades deben ser desechados a la hora de establecer sistemas mundiales en distintos ámbitos (social, informativo o económico). En una entrevista realizada en 2010 por Inter Press Service (IPS), Amartya Sen afirmaba lo siguiente: "Si no es para todos, no es globalización"16. Esta premisa deja patente que las relaciones globales solo tienen sentido cuando realmente lo son.

Una actuación mundial basada en la coherencia y la compenetración, en la tolerancia y la justicia, en el respeto y la igualdad de condiciones, en la participación libre de los ciudadanos y la comunicación, es el camino que mejor se adapta a las distintas realidades mundiales. Estas líneas permitirían que cualquier intercambio que se produjera se tradujese en un beneficio común que implicara a todos sus miembros de forma equitativa. Solo así se podría hablar de un desarrollo pleno para todos y tendría sentido el concepto de sociedad mundial.

\section{LA SOCIEDAD COMO CENTRO, EL INDIVIDUO COMO ESENCIA}

Los fenómenos y procesos globales están afectando enormemente al desarrollo de las sociedades y de los individuos que la forman. Cuando se establecen las líneas sobre las que se dibuja el desarrollo, se parte de la idea de que compartimos espacios comunes que nos impulsa a relacionarnos en todos los ámbitos. Esta percepción queda reflejada en los análisis que hace el Programa de Naciones Unidas para el Desarrollo (PNUD) a la hora de plasmar los componentes del desarrollo y los dibuja dentro de un "planeta compartido"17.

Por ello, proceder de forma global, teniendo en cuenta la actuación múltiple en zonas diversas y distantes, puede llevar a dos vías de actuación: unificar comportamientos o potenciar la diversidad. La necesidad de establecer pautas de conducta o

15 El concepto "brecha digital" comienza a utilizarse a finales de la década de los 70, cuando se inicia el proyecto Minitel en Francia, proyecto que pretendía ofrecer directorios telefónicos electrónicos. Se pretendía ofertarlos de forma gratuita para que todo el mundo pudiera adquirirlos y no quedara ningún sector de la población relegado. Este término fue retomado en EEUU en la década de los 90 al referirse al riesgo de marginación al que se veían expuestos ciertos sectores de la sociedad al no poder acceder a los sistemas informáticos, y por ende, a la sociedad de la información y del conocimiento.

16 Entrevista realizada por Sanjay Suri, de la Agencia de noticias Inter Press Service (IPS), el 22 de diciembre de 2010, en Londres. Texto íntegro disponible en el siguiente enlace:

http://ipsnoticias.net/nota.asp?idnews=86671

17 Informe sobre Desarrollo Humano 2010. "La verdadera riqueza de las naciones: caminos al desarrollo humano", PNUD, p.24. Disponible en recurso electrónico:

http://hdr.undp.org/en/media/HDR_2010_ES_Complete.pdf 
sistemas similares para el entendimiento mutuo entre diferentes poblaciones, obliga a crear unas líneas comunes que faciliten la realización de acciones que puedan ser útiles para el conjunto. Sin embargo, un empleo abusivo de formas excesivamente generalizadas puede provocar consecuencias negativas como la pérdida de identidad, aspecto esencial que vincula a los individuos entre ellos y con su sociedad.

Cuando gobiernos o instituciones llevan a cabo interacciones globales, es importante tener presente lo que Mattelart señalaba (2006:24) sobre la existencia de dos tipos de relaciones paralelas: las que se producen entre las sociedades y las que se producen entre los individuos que las forman. Este tipo de relaciones se dan en el mismo espacio, pero en distintos niveles: por un lado se desarrollan relaciones contractuales dentro de las comunidades, en los grupos primarios, y se traducen en relaciones afectivas y relaciones existenciales; por otro lado, dentro de las sociedades se crean vínculos basados en normas, reglas o leyes (elegidas por sus miembros si se trata de un sistema democrático), y se establecen relaciones impersonales, anónimas y competitivas, lo que no significa que tengan que ser exclusivas o destructivas. Es desde esta última perspectiva desde la que se parte a la hora de desarrollar interacciones a nivel mundial. Por ello, no se puede obviar la existencia ni influencia de dichas relaciones al extrapolarlas al ámbito internacional.

Si los entes públicos actúan de acuerdo a unos parámetros en los que sea fundamental el respeto e impulso de la diversidad, es posible establecer patrones globales que sean compartidos por distintas comunidades, sociedades y estados (como un mismo lenguaje visual o informático), sin perder la propia identidad. Cuando estos sistemas son llevados a la práctica, el método más eficaz para conseguir unos resultados positivos, estables y dinámicos, es la constitución de redes ${ }^{18}$ que permiten la relación y comunicación de las diversas partes de forma igualitaria y equilibrada.

La idea de red facilita el intercambio e implica la existencia de sujetos dispares que comparten líneas de interrelación; que confluyen en puntos comunes; que emplean los mismos recursos de forma equilibrada. Una red se traza en igualdad, ya que es necesaria la existencia de todos los puntos para que ésta se mantenga. La aparición de cada sujeto nuevo a la red mejora la comunicación y el alcance de las acciones globales. La red acoge e incluye, no excluye ni limita, permitiendo además una apertura mayor a la vez que una participación y vinculación entre las partes.

Uno de los paradigmas de esta idea es el establecimiento de sistemas globales de información y comunicación. El desarrollo de las nuevas tecnologías ha sido un factor clave en este proceso ya que ha permitido crear redes cada vez más estables y con mayor alcance. Este hecho ha favorecido la vinculación de zonas dispares que hasta el momento se habían visto relegadas del conjunto, abriéndoles la posibilidad de implicarse y participar de forma más activa. Diversos expertos señalaban los efectos positivos de acciones globales:

18 Al referirnos al concepto de red lo hacemos desde la perspectiva de Mattelart (2007:24-26), cuando destaca la importancia de las redes a la hora de establecer sistemas de intercambio y comunicación entre distintas sociedades y territorios. Habla de ellas como las creadoras de un "vínculo universal", alejándose de la concepción técnica del término y centrándose en el concepto de "redes sociales" a través de las que es posible "entrelazar el universo". 
De lo que se trata principalmente es de cómo dar buen uso a los notables beneficios del intercambio económico y del progreso tecnológico en una forma que preste la atención debida a los intereses de los desposeídos y desvalidos (Kliksberg y Sen, 2007:18).

Sin embargo, el uso que se ha hecho de las nuevas tecnologías en ocasiones no ha sido el esperado. La escasez de recursos o el desconocimiento de lenguajes empleados de forma universal para emplear estas tecnologías por parte de zonas con menor nivel de IDH, limita las posibilidades de participación e interconexión de sus miembros. Podemos encontrar ejemplos de ello en los informes presentados por el PNUD:

\section{Ilustración 1.}

Número de personas con acceso a Internet y abonados telefónicos por cada 100 habitantes, según nivel de desarrollo humano, 2000-2008 19

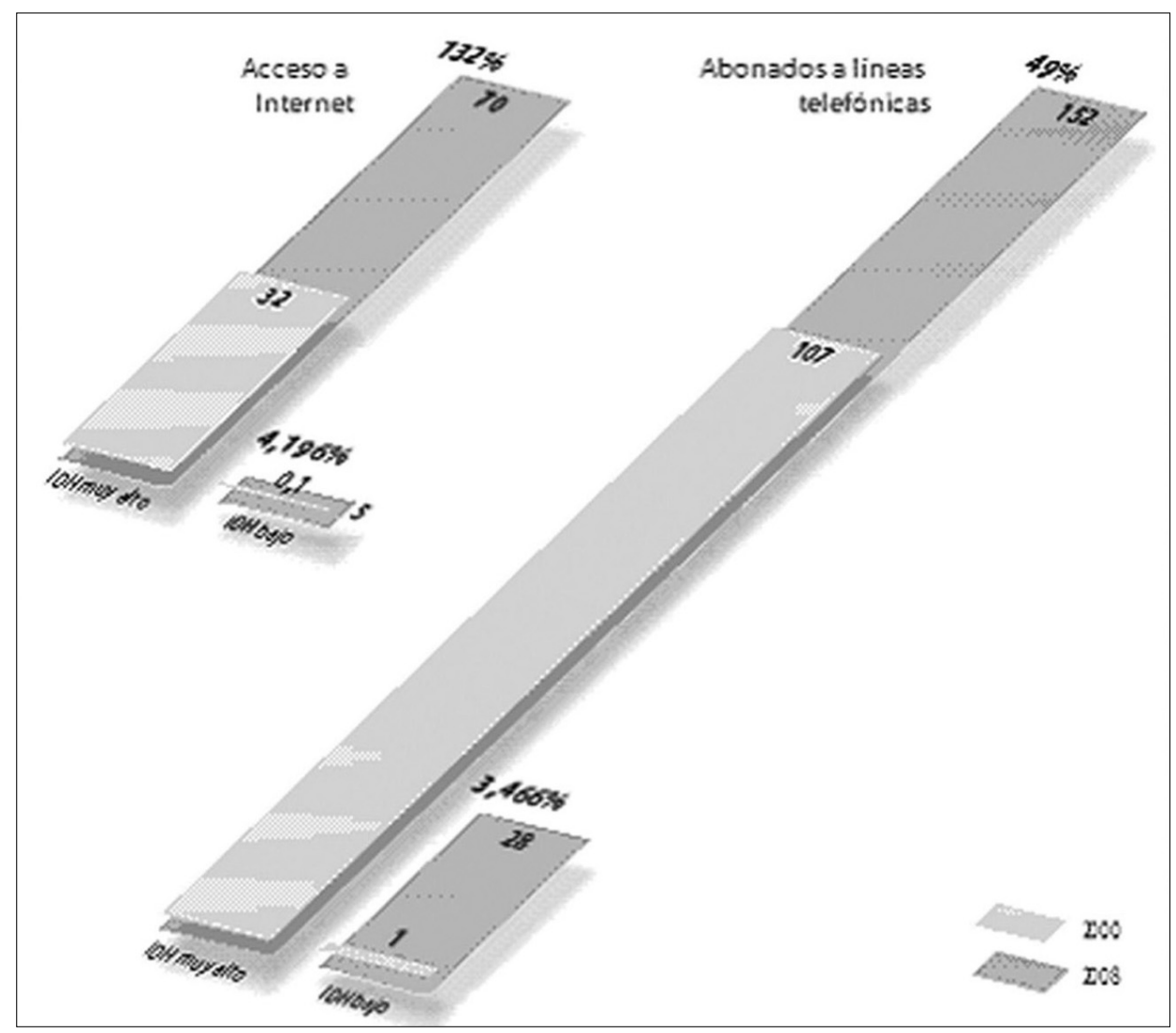

Nota: las cifras sobre las barras muestran el porcentaje de crecimiento en el período. Los abonados telefónicos incluyen líneas fijas y móviles.

Fuente: Unión Internacional de Telecomunicaciones, 2009 
Esta situación ha derivado en la imposición de los modus operandi de unas zonas sobre otras, lo que hace que determinadas sociedades se encuentren en un estado de dependencia y no puedan desarrollar sistemas influyentes propios. De esta manera, los individuos que forman parte de las distintas sociedades no ven representados sus intereses ni sus necesidades, por lo que solucionar los problemas que les afectan se complica en extremo.

Teniendo en cuenta estos aspectos, los individuos y sociedades pasan a ser protagonistas de su propio desarrollo logrando así su empoderamiento, teniendo voz pública cuando ésta quedara diluida entre el conjunto social a través de procesos democráticos. El PNUD defiende que "las personas son a la vez beneficiarias y agentes motivadores del desarrollo humano, como individuos y colectivamente." 20 De acuerdo a esta afirmación, el desarrollo humano se compone de tres factores: por un lado "bienestar" (que permite ampliar las libertades reales de las personas), por otro "empoderamiento" (que facilita las acciones de los individuos y los grupos) y por último "justicia" (que favorece la equidad respetando los derechos humanos). De esta forma, las personas empoderadas son capaces de producir cambios en su vida familiar, social y global.

Desde esta perspectiva, cabe plantearse en qué forma el individuo, con capacidad de decisión y de participación en la vida pública, podría construir una sociedad que cubriera sus necesidades. Para lograrlo necesita las herramientas que le ofrecen los medios como intermediarios entre el individuo como parte de la sociedad y las instituciones políticas y decisorias de cualquier estado de derecho.

Cuando esta interrelación entre el individuo y su sociedad se extrapola al ámbito internacional, los sujetos pasan a ser sociedades y las sociedades pasan a ser estados. La complejidad que adquieren estas interrelaciones lleva en ocasiones a que los intereses de las sociedades se vean sometidos al de los estados, y por lo tanto, a que prevalezca la idea de bienestar generalizado (en conjunto, de la mayoría) más que la de bienestar común (o de todos sus miembros). Para que esto no se produzca es necesario concebir el conjunto global sin perder la perspectiva de las partes que lo forman, o como Mattelart apunta al hablar en términos de mundialización, "pensar globalmente, actuar localmente" (2007:80), ya que cualquier medida tomada en un sentido u otro afectará irremediablemente al conjunto y a las partes. De esta idea está en consonancia con el sentido de la "circularidad global/local" (2006:28), esencial para comprender los efectos que pueden provocar acciones locales en el conjunto y viceversa. Es así como cualquier acción que se realice a nivel nacional dentro de las políticas públicas en el ámbito de la educación, la reforma agraria o la sanidad, influyen directamente en las operaciones que los distintos países realicen a nivel mundial.

Las diferencias preexistentes entre las diversas sociedades hacen que vayan surgiendo necesidades dispares que no pueden ser solventadas con la aplicación de las mismas medidas. Homogenizar situaciones que pertenecen a culturas o estructuras sociales diferentes desestabiliza los pilares en los que cada una se asienta y pervierte sus fundamentos.

20 Ibíd., p.24 
No obstante, las diferencias no tienen que entenderse como un problema o como una barrera infranqueable frente a la creación de un sistema conjunto a través del que se permita un fortalecimiento global, sino como elementos autóctonos que enriquecen el conjunto y que tienen la posibilidad de imbricarse entre ellos. Cuando distintas sociedades, gobiernos o pueblos plantean un proyecto común, éste no tiene porqué ser el resultado homogéneo de una adaptación; el proceso de colaboración y compenetración de entes diferentes lleva a la concepción de un todo complejo y completo. El conocimiento global se basa en la construcción acumulativa y la incorporación de ideas. Nunca se parte de cero. El desarrollo siempre es conjunto y complementario, además de inclusivo. Los avances se producen gracias y a partir de los realizados anteriormente. Dicha construcción, se podría visualizar como un sistema complejo con una base común y sólida con diversas ramificaciones orientadas en múltiples direcciones, que pueden ser o no coincidentes, pero que compartirían determinados asuntos globales. Es lo que David Held denomina "comunidades de destino solapadas"21 (2005:144).

Para que este conjunto tenga coherencia es necesario que se promueva la comunicación de forma mundial. La interconexión que se da entre las distintas sociedades, y que se ve incrementada en determinados ámbitos, es posible gracias al desarrollo que han sufrido las estructuras, tecnologías, herramientas y sistemas comunicativos. Este hecho reporta beneficios a nivel comercial y político, pero también, a nivel social como puede ser la apertura de las distintas culturas gracias a la implantación de sistemas de comunicación que reducen o eliminan barreras geográficas permitiendo realizar un intercambio informativo. Este tipo de relaciones externas potencia y aumenta las capacidades propias. Sin embargo, además de dar a conocer los avances producidos por cada sociedad aumentando así su presencia internacional, también se exteriorizan sus problemas, permitiendo encontrar soluciones conjuntas más eficaces, basadas en la experiencia global.

El conflicto surge cuando las relaciones de intercambio entre diversas zonas se producen de forma desigual y el desequilibrio se apodera de las sociedades. Los principales y últimos perjudicados son siempre las pequeñas sociedades y los individuos. Los grandes conjuntos camuflan la situación real de pequeños grupos bajo cifras que homogenizan situaciones dispares, logrando obtener resultados aparentemente satisfactorios para todos. Sin embargo, si se profundiza en las distintas realidades sociales se puede observar cómo se crean injusticias en nombre del "conjunto". Los errores cometidos en este ámbito han perjudicado los logros obtenidos por acciones globales. Los avances producidos en los sistemas de información y comunicación son un claro ejemplo de estas mejoras. Conseguir que exista una interco-

21 Held (2005) indica que existen varios tipos de asuntos: por un lado, los que se refieren a aspectos como la vivienda o la educación que, aunque también se defienden desde organismos supranacionales para favorecer su cumplimiento, deben ser tratados y gestionados por las diversas políticas de cada país de acuerdo a la legislación que cada uno de ellas tenga; por otro lado, existen aspectos más relacionados con la salud mundial, la economía global o el medio ambiente, que afectan a la sociedad de forma general y que, por tanto, deben ser abordados por instituciones que se sitúen por encima de posiciones políticas o sistemas gubernamentales. Sólo de esta forma se adquirirá la libertad de actuación plena necesaria para promover una equiparación de derechos y obligaciones a nivel mundial. 
municación mundial es un efecto de la necesidad de relación entre las distintas poblaciones. Por ello, al establecer las líneas que deben regir este tipo de relaciones, hay que tener presente que deben respetarse los derechos de los individuos y sus intereses también deben mantenerse en el conjunto. Una colaboración global no significa una eliminación de las partes implicadas en el intercambio, al contrario. La presencia no latente sino activa de cada uno de los actores intervinientes es la única vía para evitar que determinadas sociedades queden al margen de decisiones que les afectan de forma directa.

Del mismo modo, cuando hablamos de desarrollo, el problema y las discusiones respecto a quién y cómo se deben trazar las líneas de actuación, se agravan. Para llevar a cabo un desarrollo real de los pueblos ${ }^{22}$ es necesario e imprescindible contar con ellos. Algunos de los proyectos del Programa de Naciones Unidas para el Desarrollo (PNUD), inciden precisamente en este punto: la necesidad de que el desarrollo se produzca de la mano de sus principales beneficiarios. Así, por ejemplo, el Programa Internacional para el Desarrollo de la Comunicación (PIDC) apuesta por un desarrollo autónomo que parte de las sociedades con escasos recursos y medios y que cuenta con el apoyo de otras con mayor capacidad de actuación. La idea que propone y lleva a cabo en cada uno de los proyectos que desarrolla el PIDC es que cualquier relación establecida entre los estados se dé siempre desde la igualdad y el equilibrio, sin que se produzcan imposiciones o supeditaciones. El PIDC confía en que las sociedades que buscan un mayor desarrollo de sus potencialidades son las máximas conocedoras de la forma óptima para llegar a él. En esta misma línea, el Programa de Información Para Todos (PIPT) busca realizar acciones conjuntas que permitan el acceso y empleo de la información por parte de todos de una forma igualitaria y equilibrada.

No obstante, las limitaciones existentes en lo referente a recursos materiales o al desarrollo de las propias potencialidades dificultan aún más unas relaciones equilibradas que partan de la igualdad. Collier habla de la existencia de cuatro trampas que afectan a los países empobrecidos y a las relaciones que estos mantienen: "la trampa del conflicto, la trampa de los recursos naturales, la trampa de vivir rodeado de malos vecinos y sin salida al mar, y la trampa del mal gobierno en un país pequeño" (2009:25). Estas trampas son las que marcan el ritmo y las que inciden negativamente en los procesos de desarrollo. Las grandes dificultades a las que se tienen que enfrentar estas sociedades les restan poder y presencia en las decisiones internacionales. Es así como, al realizarse desde la desigualdad, sin la existencia de unos compromisos establecidos y aceptados por ambas partes, producen brechas y agravan los problemas.

De esta forma, cuando los gobiernos plantean la realización de acciones de cooperación internacional tienen en cuenta la divergencia de escenarios existentes y las distintas necesidades. Al realizar cualquier intervención se busca la obtención de resultados que puedan ser mantenidos por las propias sociedades sin necesidad de apoyo o ayuda externa. Esta estabilidad y sostenibilidad de los proyectos es lo que lleva al verdadero desarrollo de los pueblos. Si las necesidades son extremas o la

22 Al referirnos a pueblos lo hacemos desde la perspectiva abordada y definida por Kofi Annan en la Declaración del Milenio, durante la Cumbre del Milenio, en Nueva York, el 8 de septiembre de 2000. 
complejidad de las acciones exige una colaboración más duradera y, por tanto, la creación de proyectos de largo plazo, es importante conocer hasta qué punto se pueden crear sistemas que permitan que las propias sociedades adopten las estructuras que se proponen desde fuera. En ambos casos, el conocimiento de la zona en la que se vaya a actuar debe ser pleno. No se puede realizar ninguna acción de cooperación si se desconoce la cultura o complejidad de la sociedad con la que se va a trabajar. En este proceso la comunicación es esencial, tanto dentro de las sociedades como fuera de ellas.

Desde la década de los 80 en la que se empezaron a realizar los primeros análisis en profundidad sobre la comunicación y su influencia en el desarrollo humano, gracias al Informe MacBride que dejó patente las carencias internacionales que existían en este ámbito, la comunicación se ha convertido en un punto clave en el desarrollo y la cooperación. Las poblaciones encuentran en la comunicación una forma de expresión individual (del individuo al grupo), colectiva (entre los diferentes miembros de las comunidades) y global (entre sociedades dispersas). Gracias a estos avances ha sido posible crear estructuras sólidas de intercambio -informativo, social, cultural, educativo, etc.- que apuestan por la consolidación de un mundo más completo e integrado. La idea principal de este intercambio internacional, en el que las poblaciones se desarrollan de forma conjunta, se fortalecen con el apoyo global y se completan y complementan de modo que las potencialidades de unos puedan cubrir y soportar las necesidades de los otros. Es decir, la consolidación de un desarrollo pleno y real de los pueblos. En este sentido y desde esta perspectiva es desde la que se pueden establecer conceptos como el de globalización, mundialización o sistemas globales de comunicación. A pesar de que cada uno de ellos incida en factores diferentes (económico, humano y comunicativo), es importante cuidar en extremo los aspectos sociales, ya que son el epicentro de las relaciones internacionales y su razón de ser. 


\section{REFERENCIAS BIBLIOGRÁFICAS}

ARENAL, Celestino del, (2002): "La nueva sociedad mundial y las nuevas realidades internacionales: un reto para la teoría y la política". Cursos de Derecho Internacional y Relaciones Internacionales de Vitoria-Gasteiz 2001. Bilbao: Universidad del País Vasco, 2002, pp. 17-85.

Collier, Paul, (2007): El club de la miseria. Qué falla en los países más pobres del mundo. Madrid: Turner.

CROCKER, David, (2003): "Globalización y desarrollo humano: aproximaciones éticas". Republicanismo y educación cívica: ¿Más allá del liberalismo? coord. por Jesús Conill Sancho, David A. Crocker, pp. 75-98.

HelD, David, Un pacto global, Madrid, Taurus, 2005.

Hessel, Stéphane, (2009): Hacia nuevas solidaridades. Diez diálogos sobre Cooperación al Desarrollo. Bilbao: Catarata.

KliksBerg, Bernard. y Sen, Amartya K., (2007): Primero la gente. Barcelona: Deusto.

Mattelart, Armand, (2007): La mundialización de la comunicación. Barcelona: Paidós Ibérica.

—, (2006): Diversidad cultural y mundialización. Barcelona: Paidós Ibérica.

RoBINSON, William.I., (1998) "La globalización capitalista y la transnacionalización del Estado". Trabajo presentado en el Taller Trasatlántico sobre "Materialismo histórico y la globalización" que se realizó en la Universidad de Warwick en abril.

SEN, Amartya K., (2007 ): Identidad y violencia. Madrid: Katz.

—, (2007): "Pobreza global, justicia global". Extraído del libro Identidad y violencia, de Katz Editores. Versión publicada en el suplemento El Observador del diario Perfil, Buenos Aires: 24 de junio.

- (2002): "How to Judge Globalism" ("Juicios sobre la Globalización"), en The American Prospect, Invierno , pp. A2-A6. Traducción del inglés: Ilán Semo. Revista Fractal 22, julio-septiembre, 2001, año 6, volumen VI, pp. 37-50.

SHIVA, Vandana, (2000): "La globalización del hambre: una guerra contra la naturaleza y contra los pobres", en Globalización. Revista Web Mensual de Economía, Sociedad y Cultura, mayo. Disponible en el siguiente enlace:

$\mathrm{http} / / / \mathrm{rcci}$.net/globalizacion/2000/fg126.htm

SOLER, Fernando, (2000): "Mundialización, globalización y sistema capitalista". Revista Economía, Sociedad y Cultura. 


\title{
RESUMEN
}

Para conseguir un desarrollo pleno y sostenible es necesario contemplar los aspectos sociales como referentes prioritarios. La base de cualquier sociedad radica en las relaciones de equilibrio que se mantengan entre sus miembros. En este sentido la comunicación cumple un papel esencial. Cuando esta idea es trasladada al conjunto global los aspectos económicos toman relevancia en detrimento de lo social creando graves desequilibrios. Recuperar los principios esenciales de la globalización tomando como punto de partida el bien común y al ser humano como centro es el único camino para la construcción de sociedades realmente desarrolladas.

Palabras clave: desarrollo, comunicación, sociedad global, globalización, bien común, participación social, interactuación, equilibrio, coherencia.

\begin{abstract}
To reach a full and sustainable development is essential to have social aspects like prioritary referents. The base of any society is in relationships of balance that their members have between theirs. In this sense the communication meets an essential paper. When this idea is carried to whole global society, economic aspects become more relevance than social aspects creating serious imbalances. Recovering the essential principals of the globalization, taking common welfare like starting point and the human been like center, is the one way to build a society development real.
\end{abstract}

Key words: development, communication, global society, globalization, common welfare, social participation, interaction, balance, coherence.

\section{RÉSUMÉE}

Pour obtenir un développement plein et durable il est nécessaire avoir les aspects socials comme references et priorités. La base de toute société réside dans les relations d'équilibre maintenues parmi ses membres. Dans ce sens, la communication joue un rôle clé. Quand cette idée est transférée à des questions économiques mondiales ils deviennent pertinentes au detriment des questions sociales, en créant de graves déséquilibres sociaux. Récupérer les principles essentiels de la mondialisation en prenant comme point de départ le bien commun et les êtres humains comme le centre est le seul moyen de bâtir des sociétés développées vraiment.

Mots clé: développement, communication, société mondiale, mondialisation, bien commun, participation sociale, interaction, equilibre, coherence. 